\title{
Moore-Penrose Inverse and Semilinear Equations
}

\author{
Hugo Leiva, Raúl Manzanilla \\ Yachay Tech, School of Mathematical Sciences and Information Technology, Imbabura, Ecuador \\ Email: hleiva@yachaytech.edu.ec, hleiva@ula.ve,rmanzanilla@yachaytech.edu.ec
}

How to cite this paper: Leiva, $H$. and Manzanilla, R. (2018) Moore-Penrose Inverse and Semilinear Equations. Advances in Linear Algebra \& Matrix Theory, 8, 11-17. https://doi.org/10.4236/alamt.2018.81002

Received: November 24, 2017

Accepted: January 14, 2018

Published: January 17, 2018

Copyright $\odot 2018$ by authors and Scientific Research Publishing Inc. This work is licensed under the Creative Commons Attribution International License (CC BY 4.0).

http://creativecommons.org/licenses/by/4.0/

\section{c) (i) Open Access}

\section{Abstract}

In this paper, we study the existence of solutions for the semilinear equation $A x+f(x)=b$, where $A$ is a $m \times n, m \geq n, \quad b \in \mathbb{R}^{n}$ and $f: \mathbb{R}^{m} \rightarrow \mathbb{R}^{n}$ is a nonlinear continuous function. Assuming that the Moore-Penrose inverse $A^{\mathrm{T}}\left(A A^{\mathrm{T}}\right)^{-1}$ exists ( $A^{\mathrm{T}}$ denotes the transposed matrix of $A$ ) which is true whenever the determinant of the $n \times n$ matrix $A A^{\mathrm{T}}$ is different than zero, and the following condition on the nonlinear term $f$ satisfied $\lim _{\|x\| \rightarrow \infty} \frac{\|f(x)\|}{\|x\|}=0$. We prove that the semilinear equation has solutions for all $b \in \mathbb{R}^{n}$. Moreover, these solutions can be found from the following fixed point relation $x_{b}=A^{\mathrm{T}}\left(A A^{\mathrm{T}}\right)^{-1}\left(b-f\left(x_{b}\right)\right)$.

\section{Keywords}

Semilinear Equations, Moore-Penrose Inverse, Rothe's Fixed Point Theorem

\section{Introduction}

This work is devoted to study the existence of solutions for the following semilinear equation

$$
A x+f(x)=b, b \in \mathbb{R}^{n}, x \in \mathbb{R}^{m}
$$

where $A$ is a $m \times n$ matrix, $m \geq n, b \in \mathbb{R}^{n}$ and $f: \mathbb{R}^{m} \rightarrow \mathbb{R}^{n}$ is a nonlinear continuous function.

Definition 1.1. The Equation (1.1) is said to be solvable if for all $b \in \mathbb{R}^{n}$ there exists $x \in \mathbb{R}^{m}$ such that

$$
A x+f(x)=b
$$


Proposition 1.1. The Equation (1.1) is solvable if, and only if, the operator $A+f(\cdot): \mathbb{R}^{m} \rightarrow \mathbb{R}^{n}$ is surjective.

The corresponding linear equation $A x=b$ has been studied in [1] where a generalization of Cramer's Rule is given applying the Moore-Penrose inverse $A^{+}=A^{\mathrm{T}}\left(A A^{\mathrm{T}}\right)^{-1}$ that can be used when $\left(A A^{\mathrm{T}}\right)^{-1}$ exists, and a result from [2]. More information about the Moore-Penrose inverse can be found in [3] and [4].

In this paper, using Moore-Penrose inverse $A^{+}$and the Rothe's Fixed Theorem [5] [6] [7], we shall prove the following theorem:

Theorem 1.1. If $A^{\mathrm{T}}\left(A A^{\mathrm{T}}\right)^{-1}$ exists and $f$ is continuous and satisfies the condition

$$
\lim _{\|x\| \rightarrow \infty} \frac{\|f(x)\|}{\|x\|}=0
$$

then Equation (1.1) is solvable.

Moreover, for each $b \in \mathbb{R}^{n}$ there exists $x_{b} \in \mathbb{R}^{m}$ such that

$$
A x_{b}+f\left(x_{b}\right)=b \text {, }
$$

where $x_{b}=A^{\mathrm{T}}\left(A A^{\mathrm{T}}\right)^{-1}\left(b-f\left(x_{b}\right)\right)$.

The following theorem will be used to prove our main result.

Theorem 1.2. (Rothe's Fixed Theorem [4] [5] [6]) Let E be a Banach space. Let $B \subset E$ be a closed convex subset such that the zero of $E$ is contained in the interior of $B$. Let $\Phi: B \rightarrow E$ be a continuous mapping with $\Phi(B)$ relatively compact in $E$ and $\Phi(\partial B) \subset B$. Then there is a point $x^{*} \in B$ such that $\Phi\left(x^{*}\right)=x^{*}$.

\section{Proof of the Main Theorems}

In this section we shall prove the main results of this paper, Theorem 1.1, formulated in the introduction of this paper, which concern with the solvability of the semilinear Equation (1.1).

Proof of Theorem 1.1. Using the Moore-Penrose inverse we define the operator $\mathcal{K}: \mathbb{R}^{m} \rightarrow \mathbb{R}^{m}$ by

$$
\mathcal{K}(x)=A^{\mathrm{T}}\left(A A^{\mathrm{T}}\right)^{-1}(b-f(x)),
$$

and from condition (1.2) we obtain that

$$
\lim _{\|x\| \rightarrow \infty} \frac{\|\mathcal{K}(x)\|}{\|x\|}=0 .
$$

Claim. The operator $\mathcal{K}$ has a fixed point. In fact, for a fixed $0<\rho<1$, there exists $R>0$ big enough such that

$$
\|\mathcal{K}(x)\| \leq \rho\|x\|,\|x\|=R .
$$

Hence, if we denote by $B(0, R)$ the ball of center zero and radius $R>0$, we get that $\mathcal{K}(\partial B(0, R)) \subset B(0, R)$. Since $\mathcal{K}$ is compact and maps the sphere $\partial B(0, R)$ into the interior of the ball $B(0, R)$, we can apply Rothe's fixed point Theorem 1.2 to ensure the existence of a fixed point $x_{b} \in B(0, R) \subset \mathbb{R}^{m}$ such 
that

$$
x_{b}=\mathcal{K}\left(x_{b}\right) .
$$

Then,

$$
x_{b}=A^{\mathrm{T}}\left(A A^{\mathrm{T}}\right)^{-1}\left(b-f\left(x_{b}\right)\right) .
$$

Then

$$
A x_{b}=b-f\left(x_{b}\right) \Leftrightarrow A x_{b}+f\left(x_{b}\right)=b .
$$

This complete the proof.

From Banach Fixed Point Theorem it is easy to prove the following theorem that we will use to prove the next result of this paper.

Theorem 2.1. Let $W$ be a Hilbert space and $H: W \rightarrow W$ is a Lipschitz function with a Lipschitz constant $0<h<1$ and consider $F(w)=w+H w$. Then $F$ is an homeomorphism whose inverse is a Lipschitz function with a Lipschitz constant $(1-h)^{-1}$.

Theorem 2.2. If the Moore-Penrose $A^{\mathrm{T}}\left(A A^{\mathrm{T}}\right)^{-1}$ exists and the following condition holds

$$
\left\|f\left(x_{2}\right)-f\left(x_{1}\right)\right\| \leq L\left\|x_{2}-x_{1}\right\|, \quad x_{1}, x_{2} \in \mathbb{R}^{m},
$$

and

$$
\left\|A^{\mathrm{T}}\left(A A^{\mathrm{T}}\right)^{-1}\right\| L<\rho<1,
$$

then the Equation (1.1) is solvable and a solution of it is given by

$$
x_{b}=A^{\mathrm{T}}\left(A A^{\mathrm{T}}\right)^{-1}(I+f \circ \Gamma)^{-1}(b),
$$

where $\Gamma=A^{\mathrm{T}}\left(A A^{\mathrm{T}}\right)^{-1}$.

Proof. Define the operator $F=A+f: \mathbb{R}^{m} \rightarrow \mathbb{R}^{n}$. Then $F \circ \Gamma=I+f \circ \Gamma$ and

$$
\left\|(f \circ \Gamma)\left(b_{2}\right)-(f \circ \Gamma)\left(b_{1}\right)\right\| \leq\|\Gamma\| L\left\|b_{2}-b_{1}\right\|, \forall b_{1}, b_{2} \in \mathbb{R}^{n},
$$

and from condition (2.6)

$$
\|\Gamma\| L<\rho<1 .
$$

Therefore, from Theorem 2.1 and (2.8) we have that $F \circ \Gamma=I+f \circ \Gamma$ is a homeomorphism Lipschitizian with a Lipschitz constant $\frac{1}{1-\rho}$.

Then,

$$
F \circ\left(\Gamma \circ(I+f \circ \Gamma)^{-1}\right)=I .
$$

Hence, $x_{b}=\left(\Gamma \circ(I+f \circ \Gamma)^{-1}\right)(b)$ is a solution of (1). In fact,

$$
F\left(x_{b}\right)=b \Leftrightarrow A x_{b}+f\left(x_{b}\right)=b,
$$

and this complete the proof.

\section{Practical Example}

Now, we shall apply Theorem 1.1 to find one solution of the following semili- 
near system

$$
\left\{\begin{array}{l}
x_{1}+x_{2}+\sin \left(x_{1} x_{2}\right)=1 \\
-x_{1}+x_{2}+x_{3}+\cos \left(x_{2} x_{3}\right)=1
\end{array}\right.
$$

In this case, the vector of unknown $x$, the operators $A, f(x)$ and the system second member $b$ are:

$$
x=\left(\begin{array}{l}
x_{1} \\
x_{2} \\
x_{3}
\end{array}\right), A=\left(\begin{array}{ccc}
1 & 1 & 0 \\
-1 & 1 & 1
\end{array}\right) \text { and } f(x)=\left(\begin{array}{c}
\sin \left(x_{1} x_{2}\right) \\
\cos \left(x_{2} x_{3}\right)
\end{array}\right), b=\left(\begin{array}{l}
1 \\
1
\end{array}\right)
$$

Therefore, (3.9) can be written in the form of (1.1).

$$
A x+f(x)=b
$$

Applying Theorem 1.1 a solution of (3.10) can be obtained as a solution of the fixed-point problem:

$$
x=A^{\mathrm{T}}\left(A A^{\mathrm{T}}\right)^{-1}(b-f(x))
$$

In this particular example, one has:

$$
A^{\mathrm{T}}\left(A A^{\mathrm{T}}\right)^{-1}=\left(\begin{array}{cc}
\frac{1}{2} & -\frac{1}{2} \\
\frac{1}{2} & \frac{1}{2} \\
0 & \frac{1}{2}
\end{array}\right)
$$

To solve this problem numerically, one uses fixed-point iterations directly, i.e. one uses the following fixed point method:

$$
\left\{\begin{array}{l}
l x_{n+1}=A^{\mathrm{T}}\left(A A^{\mathrm{T}}\right)^{-1}\left(b-f\left(x_{n}\right)\right) \\
x_{0}=\left(\begin{array}{c}
20 \\
10 \\
-1
\end{array}\right)
\end{array}\right.
$$

and an error tolerance of $10^{-10}$, where the error is defined for each iteration as

$$
\operatorname{Error}(n)=\left\|x_{n}-x_{n-1}\right\| \text {, for } n=1,2, \ldots
$$

In the following figures one shows the convergence process to obtain the approximate solution. Thus, Figure 1 shows the fixed-point iterations (3.13) for different groups of iterations, i.e. in the subfigure "Iteration from 0 to 7 " it being showed the seven first fixed-point iteration values and the initial condition $x_{0}$, thus in the figure "Iteration from 8 to 15 " it being showed the next eight the fixed-point iteration values and so on for the other subfigures. By changing the scale in the subfigures, one observes the accumulation of the point-fixed iteration values in a specific place of space and that is an indicative of fixed-point iterations convergence.

As in the previous figure, Figure 2 shows the convergence error (3.14) of the fixed-point iterations for different groups of iterations. Herein, one can appreciate error convergences to zero quickly. 

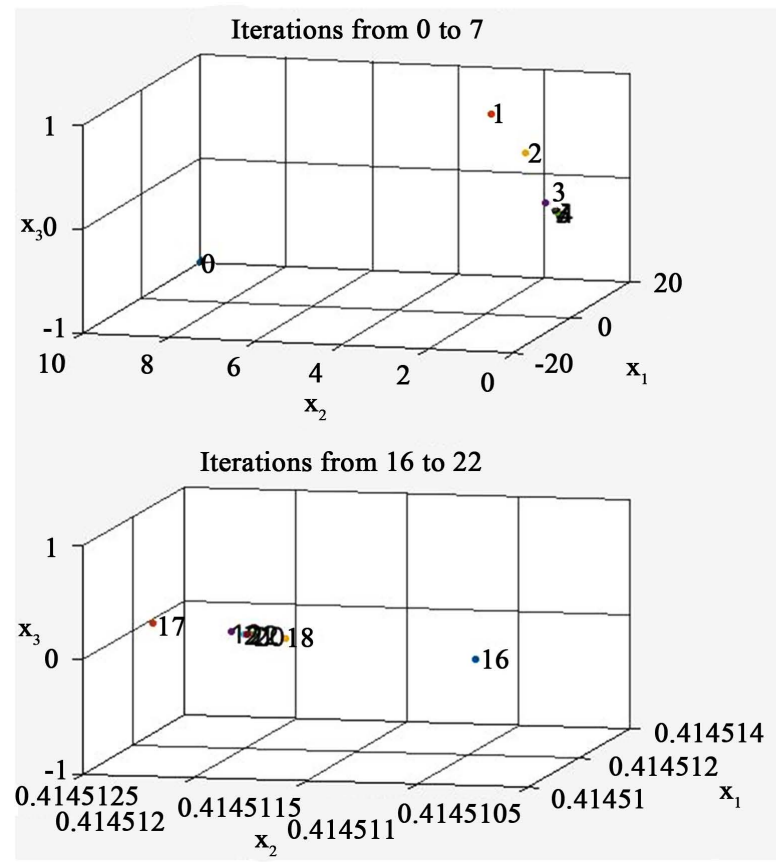

Figure 1. Convergence of fixed-point iterations.
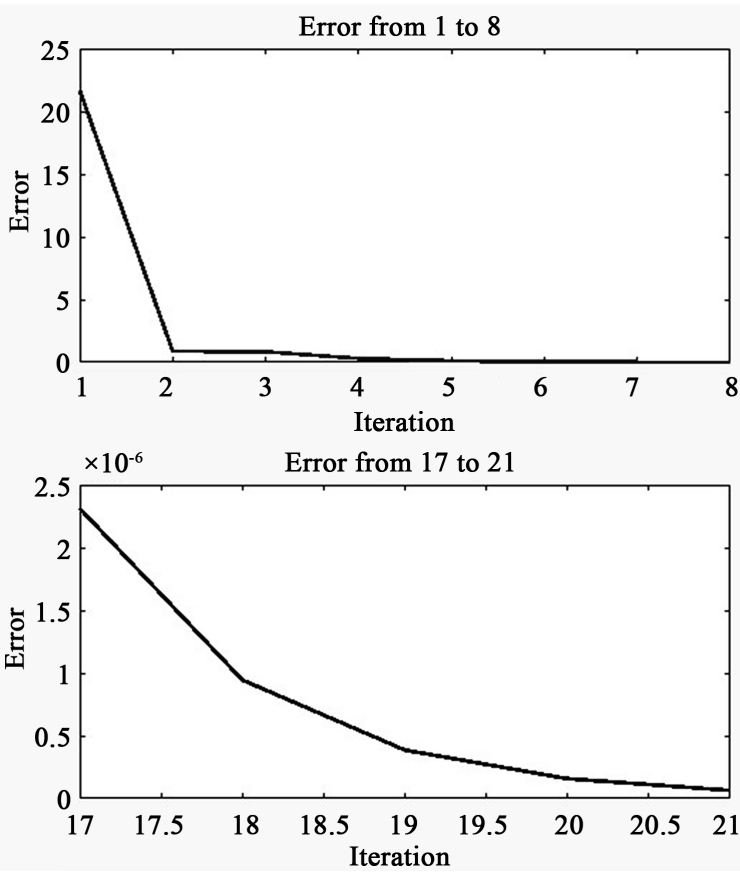

Figure 2. Error for each iteration.
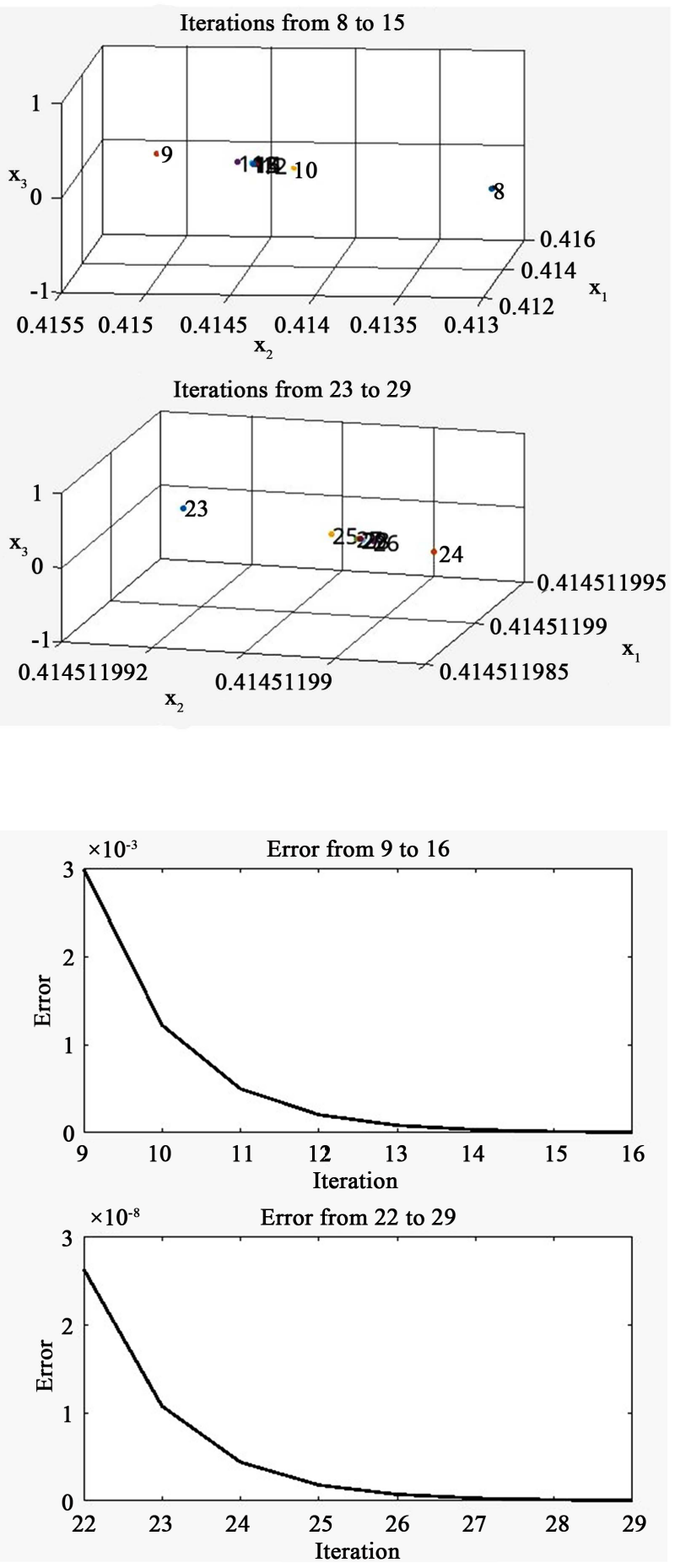
Table 1. Fixed-point iteration values.

\begin{tabular}{|c|c|c|c|}
\hline$i$ & $W_{1}$ & $W_{2}$ & $W_{3}$ \\
\hline 0 & $20.0000000000000 \mathrm{e}+000$ & $10.0000000000000 \mathrm{e}+000$ & $-1.00000000000000 \mathrm{e}+000$ \\
\hline 1 & $17.1128840687711 \mathrm{e}-003$ & $1.85618441314522 \mathrm{e}+000$ & $919.535764538226 \mathrm{e}-003$ \\
\hline 2 & $-83.6859033311723 e-003$ & $1.05192657611689 \mathrm{e}+000$ & $567.806239724032 \mathrm{e}-003$ \\
\hline 3 & $457.390133251325 \mathrm{e}-003$ & $630.527636166526 \mathrm{e}-003$ & $86.5687514576005 \mathrm{e}-003$ \\
\hline 4 & $357.047377906710 \mathrm{e}-003$ & $358.536714067085 \mathrm{e}-003$ & $744.668080187583 \mathrm{e}-006$ \\
\hline 5 & $436.167364616208 \mathrm{e}-003$ & $436.167400258264 \mathrm{e}-003$ & $17.8210279311308 \mathrm{e}-009$ \\
\hline 6 & $405.451739883687 \mathrm{e}-003$ & $405.451739883687 \mathrm{e}-003$ & $0.00000000000000 \mathrm{e}+000$ \\
\hline 7 & $418.174158181806 \mathrm{e}-003$ & $418.174158181806 \mathrm{e}-003$ & $0.00000000000000 \mathrm{e}+000$ \\
\hline 8 & $413.010123030581 \mathrm{e}-003$ & $413.010123030581 \mathrm{e}-003$ & $0.00000000000000 \mathrm{e}+000$ \\
\hline 9 & $415.124320120457 \mathrm{e}-003$ & $415.124320120457 \mathrm{e}-003$ & $0.00000000000000 \mathrm{e}+000$ \\
\hline 10 & $414.261735970446 \mathrm{e}-003$ & $414.261735970446 \mathrm{e}-003$ & $0.00000000000000 \mathrm{e}+000$ \\
\hline 11 & $414.614167222791 \mathrm{e}-003$ & $414.614167222791 \mathrm{e}-003$ & $0.00000000000000 \mathrm{e}+000$ \\
\hline 12 & $414.470255524096 \mathrm{e}-003$ & $414.470255524096 \mathrm{e}-003$ & $0.00000000000000 \mathrm{e}+000$ \\
\hline 13 & $414.529034297305 \mathrm{e}-003$ & $414.529034297305 \mathrm{e}-003$ & $0.00000000000000 \mathrm{e}+000$ \\
\hline 14 & $414.505029226087 \mathrm{e}-003$ & $414.505029226087 \mathrm{e}-003$ & $0.00000000000000 \mathrm{e}+000$ \\
\hline 15 & $414.514833210466 \mathrm{e}-003$ & $414.514833210466 \mathrm{e}-003$ & $0.00000000000000 \mathrm{e}+000$ \\
\hline 16 & $414.510829199791 \mathrm{e}-003$ & $414.510829199791 \mathrm{e}-003$ & $0.00000000000000 \mathrm{e}+000$ \\
\hline 17 & $414.512464474425 \mathrm{e}-003$ & $414.512464474425 \mathrm{e}-003$ & $0.00000000000000 \mathrm{e}+000$ \\
\hline 18 & $414.511796615081 \mathrm{e}-003$ & $414.511796615081 \mathrm{e}-003$ & $0.00000000000000 \mathrm{e}+000$ \\
\hline 19 & $414.512069374520 \mathrm{e}-003$ & $414.512069374520 \mathrm{e}-003$ & $0.00000000000000 \mathrm{e}+000$ \\
\hline 20 & $414.511957977294 \mathrm{e}-003$ & $414.511957977294 \mathrm{e}-003$ & $0.00000000000000 \mathrm{e}+000$ \\
\hline 21 & $414.512003472857 \mathrm{e}-003$ & $414.512003472857 \mathrm{e}-003$ & $0.00000000000000 \mathrm{e}+000$ \\
\hline 22 & $414.511984892088 \mathrm{e}-003$ & $414.511984892088 \mathrm{e}-003$ & $0.00000000000000 \mathrm{e}+000$ \\
\hline 23 & $414.511992480630 \mathrm{e}-003$ & $414.511992480630 \mathrm{e}-003$ & $0.00000000000000 \mathrm{e}+000$ \\
\hline 24 & $414.511989381406 \mathrm{e}-003$ & $414.511989381406 \mathrm{e}-003$ & $0.00000000000000 \mathrm{e}+000$ \\
\hline 25 & $414.511990647155 \mathrm{e}-003$ & $414.511990647155 \mathrm{e}-003$ & $0.00000000000000 \mathrm{e}+000$ \\
\hline 26 & $414.511990130213 \mathrm{e}-003$ & $414.511990130213 \mathrm{e}-003$ & $0.00000000000000 \mathrm{e}+000$ \\
\hline 27 & $414.511990341336 \mathrm{e}-003$ & $414.511990341336 \mathrm{e}-003$ & $0.00000000000000 \mathrm{e}+000$ \\
\hline 28 & $414.511990255112 \mathrm{e}-003$ & $414.511990255112 \mathrm{e}-003$ & $0.00000000000000 \mathrm{e}+000$ \\
\hline 29 & $414.511990290326 \mathrm{e}-003$ & $414.511990290326 \mathrm{e}-003$ & $0.00000000000000 \mathrm{e}+000$ \\
\hline
\end{tabular}

\section{Supported}

This work has been supported by Yachay Tech University.

\section{References}

[1] Leiva, H. (2015) A Generalization of Cramer's Rule. Linear Algebra and Matrix Theory, 5, 156-166. https://doi.org/10.4236/alamt.2015.54016 
[2] Burgstahier, S. (1983) A Generalization of Cramer's Rule. The Two-Year College Mathematics Journal, 14, 203-205. https://doi.org/10.2307/3027088

[3] Ben-Israel, A. and Greville, T.N.E. (2002) Generalized Inverses: Theory and Applications. Springer-Verlag, Berlin.

[4] Campbell, S.L. and Meyer, C.D. (1979) Generalized Inverses of Linear Transformations. Dover Publ, New York.

[5] Banas, J. and Goebel, K. (1980) Measures of Noncompactness in Banach Spaces. Lecture Notes in Pure and Applied Mathematics, 60. Marcel Dekker, Inc., New York.

[6] Isac, G. (2004) On Rothe's Fixed Point Theorem in General Topological Vector Space. Analele Stiintifice ale Universitatii Ovidius Constanta, 12, 127-134.

[7] Smart, J.D.R. (1974) Fixed Point Theorems. Cambridge University Press, Cambridge. 